\title{
Arterialización venosa temporal del pie diabético
}

\author{
Temporal venous arterialization of the diabetic foot \\ Francisco Lengua, Andrés La Madrid, Carlos Acosta, Jorge Vargas*
}

\section{Resumen}

Contexto: La primera idea que tuvieron los cirujanos (1902) para evitar amputaciones por isquemia fue la de desviar el flujo arterial al sistema venoso por intermedio de una fístula arteriovenosa entre vasos adyacentes, pero con resultados inciertos. Desde entonces se han inventado las simpatecomias, las endarteriectomias y los injertos puentes o bypass $\mathrm{y}$, últimamente, otros avances médicos, quirúrgicos y endovasculares. Sin embargo, en el mundo se siguen haciendo amputaciones, sobre todo en diabéticos. La arterialización de las venas del pie, basada en la vieja idea de la circulación invertida, constituye una esperanza más para estos pacientes ya condenados a la pérdida del miembro.

Objetivo: Demostrar que la arterialización de las venas del pie en diabéticos con lesiones neuroisquémicas, generalmente infectadas (pie diabético), es un método eficaz y durable, aunque el puente solo funcione temporalmente.

Pacientes y método: De enero de 2000 a febrero de 2009, 59 pacientes con pie diabético fueron tratados por arterialización de las venas del pie. Un deceso precoz no ha sido contabilizado. De los 58 pacientes restantes, 44 fueron hombres y 14 mujeres, con edad promedio de 71 años: (53-91 años), 54 en estado IV de Fontaine y cuatro en estado IIIB. La arterialización fue hecha con un injerto venoso invertido anastomosado, proximalmente en una arteria con buen flujo (ilíaca externa, femorales o poplítea) distalmente en la vena marginal interna del pie, con destrucción de las válvulas de las venas del dorso.

Resultados: De los 58 arterializados, 12 fueron fracasos por trombosis precoz, siendo amputados, y 46 fueron éxitos (79\%): seis de corto, 12 de mediano y 28 de largo plazo. En los grupos que se ajustan a la definición de éxito (dos) hubo 38 trombosis tardías del puente (95\%) y cuatro recidivas de la sintomatología isquémica. No ha habido ningún caso de sobrecarga cardíaca ni de várices.

Conclusión: La arterialización en el pie diabético es posible, eficaz y durable, gracias, posiblemente, a la inducción de una neoarteriogénesis y neoangiogénesis, que mantiene los beneficios aun después de que el puente se haya ocluido (función temporal).

Palabras clave: Salvataje de miembro, pie diabético, arterialización temporal, amputación de miembro.

\section{Introducción}

A comienzos del siglo XX, la primera idea que tuvieron los cirujanos (San Martín, 1902, España, y Jaboulay, 1902, Francia) para evitar amputaciones por isquemia, fue

\begin{abstract}
Background: The first idea of surgeons (1902) to avoid amputations due to ischemia was to deviate the arterial flow to the venous system using an arteriovenous fistula between adjacent vessels; however, the results were unreliable. Since then, sympathectomies, endarterectomies and bypasses have been created, and more recently, other medical, surgi$\mathrm{cal}$, and endovascular advances have been used. However, amputations continue to be performed worldwide mainly in diabetic patients. The arterialization of the foot veins, based on the old idea of inverted blood flow, is a new possibility for these patients who, otherwise, could lose their limbs.
\end{abstract}

Objective: To demonstrate that arterialization of the foot veins in diabetic patients with neuroischemic lesions, usually infected (diabetic foot), is an effective and long-lasting method, even though the bypass only works temporally.

Patients and method: From January 2000 to February 2009, 59 patients with diabetic foot were treated by means of arterialization of the foot veins. An early death was not included in the analysis. Of the 58 remaining patients, 44 were male and 14 were female. Their mean age was 71 years old: (53-91 years). Fifty-four of them were classified as being Fontaine IV and four were IIIB. The arterialization was conducted using an inverted venous graft proximally anastomized to an artery presenting good flow (external iliac, femoral or popliteal arteries) distally in the internal marginal vein of the foot, eliminating the valves of the foot arch veins.

Results: Of the 58 patients who underwent arterialization, 12 failed due to early thrombosis, having amputated limbs, and 46 succeeded (79\%): six at short-term, 12 at medium-term, and 28 at long-term. In the groups who were considered to have a successful outcome (two), there were 38 late bypass thromboses (95\%) and four relapses of the ischemic symptoms. There was no case of heart overload or varices.

Conclusion: The arterialization of the diabetic foot is possible, effective and long-lasting probably thanks to the induction of a neoarteriogenesis and neo-angiogenesis that maintain the benefits even after the bypass has occluded (temporal function).

Key words: Limb salvation, diabetic foot, temporal arterialization, limb amputation.

la de derivar la sangre arterial al sistema venoso mediante una fístula entre vasos adyacentes. Posteriormente, dicha técnica ha sido empleada por diversos cirujanos de diferentes países pero, por lo general, ha sido abandonada de-

\footnotetext{
* Servicio de Cirugía torácica y C-V, Hospital de Policía, Lima, Perú.

No se declararon conflictos de intereses asociados a la publicación de este artículo. Artículo recibido el 06.04.09, aceptado el 13.01.10.

J Vasc Bras. 2010;9(1):14-20.

Copyright $@ 2010$ by Sociedade Brasileira de Angiologia e de Cirurgia Vascular
} 
bido a sus resultados inciertos. En diabéticos fue empleada por primera vez por Halsted y Vaugham, 1912 (EUA), pero con malos resultados.

En 1948, Kunlin (Francia) ${ }^{1}$, al haber realizado el primer puente o bypass largo con éxito, abre una nueva era en el tratamiento de la isquemia.

Las mejoras del material de sutura, de las técnicas angiográficas y el empleo de la microcirugía han permitido la realización de puentes en arterias de pequeño calibre a nivel del tobillo y del pie $^{2}$; a veces, aun con resección ósea ${ }^{3}$. Sin embargo, para un número no despreciable de pacientes está todavía hoy fuera de alcance todo recurso terapéutico convencional, tanto médico como quirúrgico ${ }^{4}$. Esos pacientes están condenados a una amputación de miembro.

Siguiendo las ideas de Leger", que afirma que "nuestras constataciones angiográficas, al contrario, prueban hasta la saciedad que la circulación se puede realizar a contracorriente en el sistema venoso" y con la ayuda del pontage de Kunlin, nosotros hemos retomado esa antigua idea, pero modificando su modo de empleo: la arterialización de las venas del pie (AVP) con puente (Figura 1). Así concebida, esta técnica fue empleada por primera vez en 1974 en tres diabéticos con resultados alentadores ${ }^{6}$. Sin embargo, ella no tuvo buena acogida y solo 20 años después ha co-

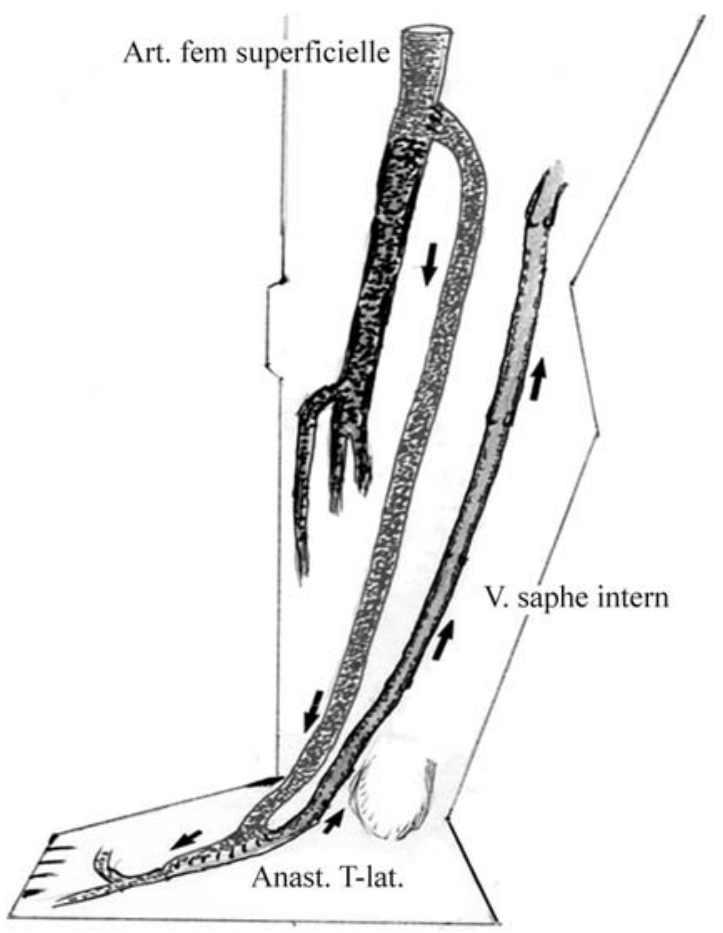

Figura 1 - Esquema de la arterialización venosa del pie menzado a ser adoptada debido a publicaciones de su empleo con buenos resultados hechas por otros equipos de cirugía vascular ${ }^{7-10}$.

Actualmente, existe la tendencia de agrupar a los diabéticos con trastornos tróficos neuroisquémicos e infectados en los pies bajo la denominación de "pie diabético" (necrosis tisulares, úlceras y gangrenas), unificando así una de las más temidas complicaciones de la diabetes melitos. Por su severidad, dichas lesiones conducen a una amputación de miembro de 10 a 20 veces más frecuentes en el diabético que en el no diabético ${ }^{11}$.

\section{Material y métodos}

Entre enero de 2000 y febrero de 2009 fueron tratados por AVP 59 pacientes con pie diabético, de los cuales uno fallece al $18^{\circ}$ día del postoperatorio, no siendo, por eso, contabilizado. De los 58 pacientes restantes, 44 fueron hombres y 14 mujeres, con edad promedio de 71 años (extremo: 53-91), siendo 50 de tipo 2 o no insulinodependiente y ocho del grupo 1 (Organización Mundial de la Salud OMS). Cincuenta y cuatro de esos pacientes se encontraban en estado IV de Fontaine y cuatro, en estado IIIB. Treinta y cinco eran hipertensos, 15 presentaban trastornos del ritmo cardíaco, 12 tenían enfermedad coronaria, ocho, insuficiencia renal (de estos, dos estaban en hemodiálisis), seis padecían de un comienzo de Parkinson, cinco presentaban secuelas de infarto del miocardio, cuatro, retinitis diabética y tres, cáncer de próstata.

La arteriografía que comprendía la totalidad del miembro mostraba un compromiso severo de las arterias de la pierna y del pie. En dos pacientes, se hizo una exploración quirúrgica antes de la arterialización: arteria pedia y tibial posterior. El sistema venoso distal ha sido evaluado clínicamente y al Doppler.

Como injerto, se ha dado la preferencia a la vena safena interna sacada de uno o de ambos miembros, según el caso.

El injerto fue en 32 casos sólo de un segmento venoso, 27 invertidos y cinco no invertidos, pero con destrucción previa de las válvulas. Fue compuesto de dos segmentos venosos en 18 casos, en ocho casos fueron mixtos [politetrafluoretileno (PTFE) más vena invertida colocada siempre distalmente], siendo seis veces secuencial (PTFE más vena y uno con vena de un familiar) y dos, mixtos directos. La arteria donante fue en 25 casos la poplítea, en 27 la fe- 
moral superficial, en tres la femoral común y en tres la arteria ilíaca externa.

La destrucción de las válvulas del pie se hizo con un juego de cinco valvulótomos flexibles, cuatro de $24 \mathrm{~cm}$ de longitud, provistos de olivas cortas de extremidad cónica implantadas en ángulo de unos 10 grados para la destrucción de las válvulas de las venas del dorso del pie, y uno pequeño de $6 \mathrm{~cm}$ con oliva implantada sin angulación para la destrucción de las válvulas ostiales de las colaterales desembocando en la flebotomía. La ruptura valvular fue hecha con un golpe seco, rotando la oliva hacia la luz vascular para evitar desgarros de la pared venosa (Figura 2).

\section{Técnica operatoria}

Se describe la realización de un puente de dos segmentos (12-14).

La anestesia de elección es la peridural con catéter. La lesión necrótica es aislada con un material estéril e impermeable. Se protege el talón (de la compresión prolongada) con un guante quirúrgico lleno con agua estéril colocado debajo del tendón del calcáneo (tendón sóleo/gemelos que se inserta en el calcáneo).

La intervención es realizada por dos equipos: el primero saca las safenas internas de ambos muslos a fin de obtener la longitud necesaria de injerto venoso, las prepara e implanta una de ellas en la arteria donante (femoral superficial u otra). Hace una incisión longitudinal oblicua en la parte baja, lado interno, de la rodilla para tunelizar la parte proximal del trayecto del puente. El segundo equipo realiza la disección de la vena marginal interna del pie. Si

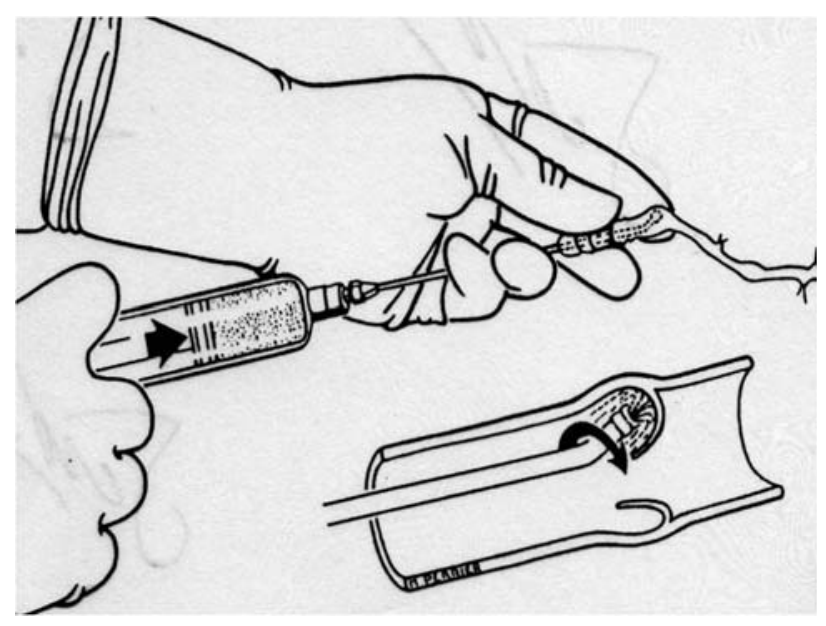

Figura 2 - Manera de destruir las válvulas del injerto para su colocación no invertida el trayecto de esta vena es visible, se hace una incisión curva con abertura superior en la fosita premaleolar a unos $10 \mathrm{~mm}$ por debajo del trayecto de esa vena para evitar que la incisión "caiga" sobre la vena misma, comprometiendo la anastomosis (necrosis cutánea). Se colocan hilos de tracción en el borde de la herida operatoria comprendiendo la piel, el tejido celular subcutáneo y la fascia dorsal superficial. En esta exposición de la vena marginal interna (así como en el cierre de la herida operatoria) está prohibido el empleo de pinzas de disección y separadores para evitar la necrosis cutánea.

Si el trayecto de la vena marginal interna no es visible, con una pequeña incisión por delante del maléolo interno se aísla un segmento de la vena safena interna premaleolar y se le pasa alrededor un hilo cuya tracción señalará el trayecto de la vena marginal interna.

Se tuneliza el trayecto del puente en la pierna en cuyo lecho se colocará una sonda de material plástico ( $\left.\mathrm{N}^{\circ} 24\right)$ por donde se pasará el injerto con facilidad y sin rotación (el tunelizador metálico no debe ser empleado por ser rígido y no adaptarse a la forma de la tibia, produciendo demasiado despegamiento de la piel con peligro de necrosis consecutiva).

Cuando el injerto está en su lugar, se retira la sonda y se talla su extremo distal para evitar la anastomosis en “bolsa". Una flebotomía de longitud según se juzgue necesaria (en relación con la disposición de las colaterales del segmento de vena marginal interna expuesto, cuyas válvulas ostiales deben ser destruidas) es hecha ligeramente hacia el borde externo de la vena marginal interna, (en relación con el eje del cuerpo) a fin de que la anastomosis quede un tanto "echada". A través de dicha flebotomía se rompen las válvulas accesibles de las venas del pie con el material diseñado para tal fin (los dilatadores arteriales solo repliegan las válvulas contra la pared) (Figura 3). Un surjet con prolene 7/0 es efectuado con lentes de aumento, salvo el ángulo distal de la anastomosis, que es hecho con puntos separados. Los dos segmentos venosos son anastomosados en término-terminal en la incisión que se hizo en la rodilla. El clampaje de la vena marginal interna y de sus colaterales se hace por tracción de un hilo delgado. Cuando existe el riesgo de compresión de la anastomosis al cierre de la herida operatoria, sus bordes pueden ser dejados entreabiertos (hasta $6 \mathrm{~mm}$ ), pero siempre se debe recubrir 


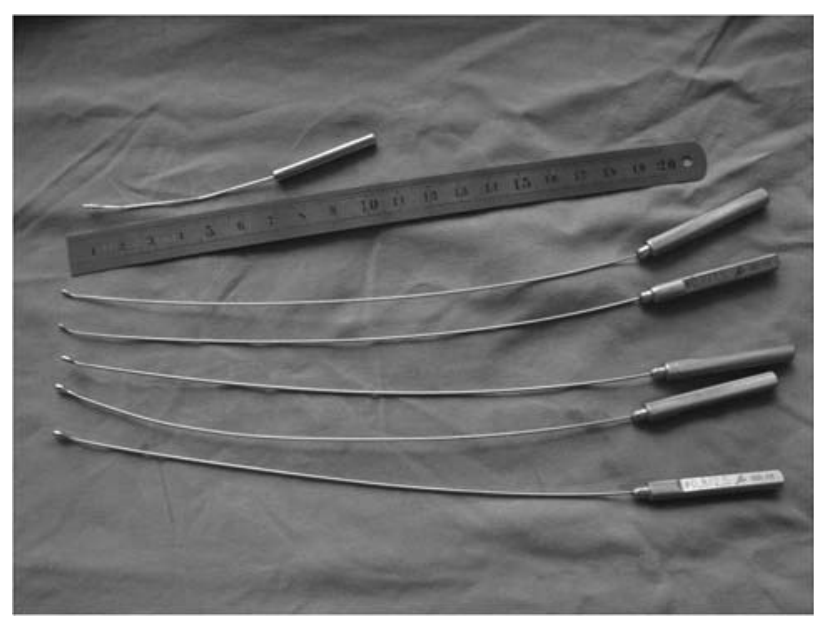

Figura 3 - Valvulótomos flexibles

con piel todo vaso expuesto con uno o dos puntos separados.

En el postoperatorio inmediato, los pacientes han recibido heparina de BPM durante una semana y luego se continúa con warfarina.

\section{Resultados}

Criterios de evaluación de los éxitos. Han sido considerados éxitos los casos en los que una amputación con pérdida del apoyo (talón) pudo ser evitada como mínimo por un año, con puente permeable por lo menos por un mes, con supresión de los dolores, cicatrización de las lesiones necróticas (con/sin amputación parcial del pie) y conservación de estos beneficios a pesar de la oclusión del puente. Asimismo, siguiendo la clasificación clásica, se han considerado tres tipos de éxitos: de corto plazo (entre 1 mes y 1 año), de mediano plazo (entre 1 y 5 años) y de largo plazo (5 años o más).

De los 58 pacientes, 12 fueron fracasos y fueron amputados a pesar de los intentos de desobstrucción, nueve supracondilias y tres infracondilias. Los otros 46 fueron éxitos (79\%), siendo seis de corto plazo, 12 de mediano plazo y 28 de largo plazo. Ajustándonos a los criterios de éxito, líneas arriba mencionados, solo serán evaluados los éxitos de mediano y largo plazo.

De los 40 éxitos de mediano y largo plazo, 38 se trombosaron (95\%), siete pacientes han fallecido (dos en el grupo de mediano y cinco en el de largo plazo) por enfermedades intercurrentes y tres pacientes fueron perdidos de vista después de un seguimiento de dos años en prome- dio. Todos los pacientes han tenido un seguimiento de 4 años y 4 meses en promedio. La duración del puente fue de 8 meses en promedio. El tiempo de cicatrización de las lesiones necróticas fue variable, dependiendo de la extensión, localización y grado de infección de las necrosis, sin embargo se puede decir que esa cicatrización se obtuvo en entre 3 y 4 meses en promedio y que en la cuarta parte de los casos se obtuvo con la ayuda de autoinjertos de piel. Dos pacientes conservan su puente permeable, uno después de 1 año y 10 meses y luego de 6 años y 10 meses el otro.

\section{Complicaciones}

En los éxitos con trombosis tardías (después de $1 \mathrm{mes}$ ), ha habido cuatro recidivas de la isquemia, de las que un paciente falleció de septicemia sin haber sido reintervenido; los otros tres casos fueron tratados con éxito con desobstrucción y angiolastía de la anastomosis distal. También se verificaron cuatro trombosis parciales en los casos con flebotomía larga $(5 \mathrm{~cm})$, de las cuales dos fueron tratadas por dilatación percutánea y las otras dos, cuyas estenosis comprendían la parte distal de la anastomosis, fueron dejadas en observación (Figura 4), habiendo conservado el miembro hasta el momento de realizar este trabajo: 3 años.

Además, se han presentado 16 necrosis cutáneas de la herida operatoria del pie sin comprometer la anastomosis, cinco casos de progresión repetida postoperatoria de las

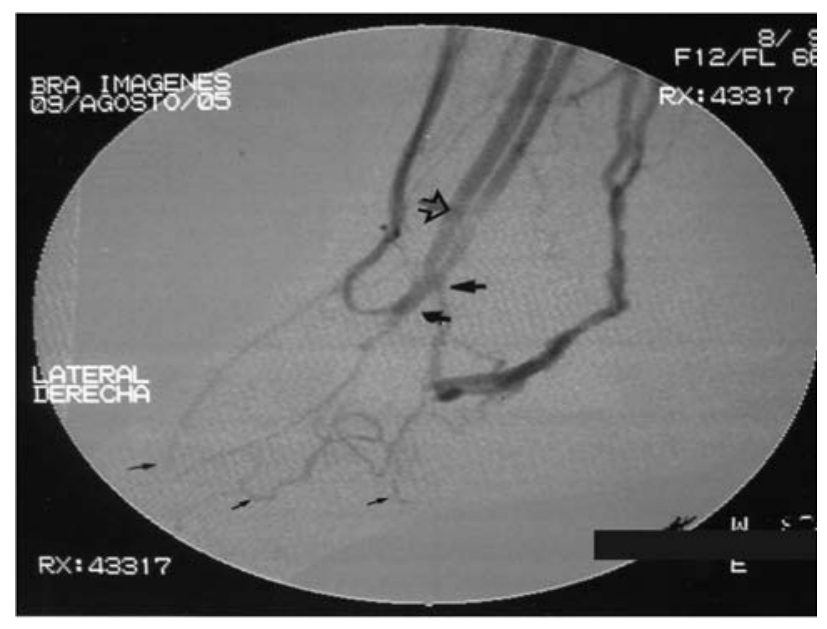

Figura 4 - Diabética de 72 años con necrosis. Control angiográfico hecho al $18^{\circ}$ día por disminución de la intensidad del pulso venoso en el pie por sospecha de trombosis parcial de la anastomosis distal. La parte proximal de ésta (dos flechas) queda funcional permitiendo la arterialización del pie (3 flechas pequeñas). La flecha hueca señala el puente que permanece permeable (control clínico) 4 años. La paciente vive 
necrosis del pie con puente permeable tratados con limpieza quirúrgica, tres que necesitaron una amputación transmetatarsiana, tres casos de síndromes necróticos dolorosos y dos de hiperestesias dolorosas desencadenadas al más leve roce de la piel, todos ellos tratados con bloqueos del simpático lumbar. Hubo tres falsas rutas sin consecuencias. No ha habido ningún caso de sobrecarga cardíaca ni de várices del miembro operado.

\section{Discusión}

De acuerdo con Leriche, que dice que "la medicina nada gana con el desprecio del hecho clínico en beneficio de anotaciones en cifras" ${ }^{\prime 15}$, nuestros resultados son dados de una manera simple, clásica, teniendo como base la observación clínica, el examen Doppler y los controles angiográficos. Además, los parámetros adoptados en la evaluación de los resultados de los puentes clásicos no son totalmente aplicables a la arterialización del pie. En efecto, si la trombosis de los puentes de arterialización se produce entre el primero e sexto mes de funcionamiento, la amputación del miembro se retarda o se evita aun a mediano plazo. Es posible que el puente de arterialización haya promovido la inducción de neocolaterales (arteriogénesis: Figuras 5 y 6) y de neoarteriolas (arteriogénesis) ${ }^{16,17}$ responsables de la mejora de las lesiones isquémicas de los pies, incluso después de la oclusión del injerto puente. En los puentes clásicos, la conservación del miembro está estrechamente ligada a la duración del puente ${ }^{18-21}$.

Toursarkissiam $^{22}$, en el análisis de 80 diabéticos operados con puente distal, ha tenido un porcentaje global de amputaciones mayor de $50 \%$, con un tiempo de permeabilidad del puente de 13 meses como promedio y un seguimiento de 14 meses después de la oclusión del puente. En nuestra serie, hemos salvado 48 miembros, con un promedio de permeabilidad del puente de 8 meses y un seguimiento promedio de 4 años y 4 meses. No ha habido ningún caso de amputación mayor. Además, de esos 80 puentes distales solo 35 (44\%) han sido hechos en las arterias por debajo de los maléolos (31 a la pedia y cuatro a las plantares). A nuestro criterio, esos puentes son los únicos que podrían ser comparados con nuestras arterializaciones del pie en un estudio randomizado. Por otro lado, en los puentes clásicos, la mayoría de estenosis han sido de presentación tardía y sobre todo en la anastomosis proximal ${ }^{23}$, mientras que en las AVP lo han sido en la anastomosis distal y de aparición más temprana. Al contrario, en la revasculariza-

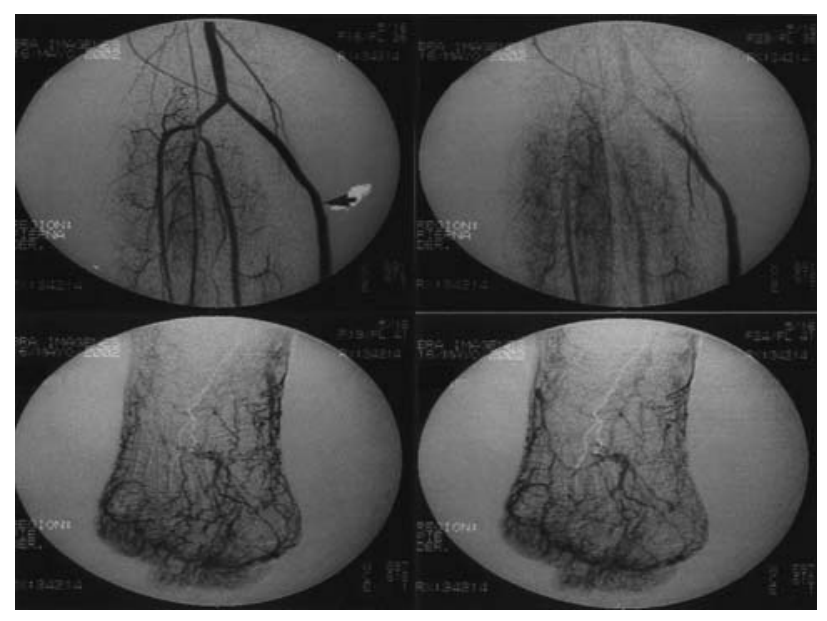

Figura 5 - Cuatro vistas. Paciente de 58 años al momento de la arterialización. Control hecho al cuarto año con el puente funcional (flecha). Imágenes superiores: el aumento del flujo sanguíneo por largo tiempo produce una dilatación de la arteria poplítea con el desarrollo de una gran cantidad de arteriolas en la pierna. Imágenes inferiores: se observa una gran cantidad de arteriolas y de vénulas (vasculogénesis) de calibre fino, con opacificación de la arcada venosa plantar superficial. El puente quedó permeable 4,5 años. Los beneficios de la operación se conservan desde entonces, a los 10 años. Fue arterializado del lado opuesto en abril de 2006. Vive
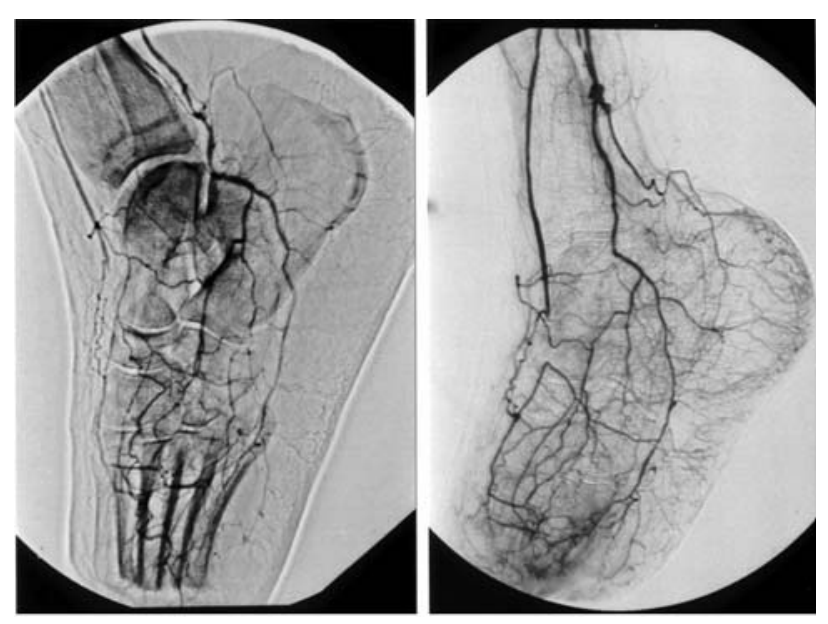

Figura 6 - Diabética de 73 años. Arteriografías hechas con la misma técnica radiológica. A) Preoperatorio (amputación transmetatarsiana); B) Control hecho 4 meses después de la operación y después de la oclusión espontánea del puente. "Bella imagen" del pie. Se visualiza un gran número de arteriolas (neoarteriogénesis). Los beneficios han sido conservados hasta el deceso, 5 años después de la operación de neoplasia digestiva

ción con puente clásico la supresión de la sintomatología isquémica ha sido rápida, puesto que ella se hace por vía fisiológica, mientras que en la AVP la misma recuperación ha sido lenta, a veces aun con progresión de las necrosis a pesar de la permeabilidad del puente, lo que se explica por 
una revascularización hecha, al menos durante cierto tiempo, por vía no fisiológica.

Mar y Ramos ${ }^{24}$, en comunicación personal, con técnica parecida a la nuestra, han obtenido $75 \%$ de éxitos en 21 arterializaciones (trabajo en vías de publicación).

Existen otras formas de arterializar las venas del pie, como la propuesta por Busato et al. ${ }^{25,26}$, quienes, en un trabajo en vías de publicación intitulado "The use of long saphenous vein 'in situ' to the arterialización of the Foot Venous Arch", hacen referencia a una serie de 16 casos arterializados con la variante que nos ocupa, con buenos resultados en la mayoría de los casos.

Los buenos resultados de las AVP en diabéticos, que en el pasado correspondían al $64 \%{ }^{12}$, con la flebotomía según necesidad y una destrucción más eficaz de las válvulas, es actualmente del 79\%. Estos resultados han sido obtenidos en pacientes considerados como inoperables con puente clásico distal.

Nosotros somos conscientes de que el trabajo adolece de falta de pruebas fehacientes de nuestros resultados, pruebas que desgraciadamente no están a nuestro alcance por falta de medios; sin embargo, los resultados de nuestro estudio son el producto de una larga observación clínica y de controles radiológicos.

\section{Conclusión}

La arterialización de las venas en el pie diabético es una alternativa más en pacientes para quienes los tratamientos médicos y quirúrgicos convencionales están fuera de alcance, siendo eficaz y durable aunque el puente solo funcione temporalmente.

\section{Referencias}

1. Kunlin J. Le traitement de la ischémie artéritique par la greffe veineuse longue. Rev Chir. 1951;206-35.

2. Pomposelli FB, Jepsen SJ, Gibbon GW, Campbell DR, Logerfo FW. Efficacy of the dorsal pedal bypass for limb salvage in diabetic patients: Short term observations; J Vasc Surg. 1990;111:745-52.

3. Ascer E, Veith F, Gupta SK. Bypasses to plantar arteries and other tibial branches. An extended approach to limb salvage. J Vasc Surg. 1988;8:434-41.

4. Campbell WB, Verfaille P, Ridler BM, Thomson JF. Non operative treatment of advanced limb ischaemia: the division for palliative care. Eur J Vasc Endovasc Surg. 2000;19:24649.

5. Leger L. Essais de revscularisation d'un membre par anastomose artério-veineuse termoino-terminal. Press Med. 1949;57:1198-200.
6. Lengua F. Technique d'artérialisation du réseau veineux du pied. Press Med. 1975;4:1039-42

7. Pokrovskii AV, Chupin DV, Khorovets AG. Arterialization of the foot venous system in the treatment of the critical limb ischaemia and distal bed occlusion. Ang Vasc Surg. 1996;4:73-93.

8. Taylor RS, Belli A, Jacob S. Distal venous arterialisation for salvage of critically ischaemic inoperable limbs. Lancet. 1999;354:1962-65.

9. Engelke C, Morgan RA, Quarmby JW, Taylor RS. Distal venous arterialization for lower limb salvage: Angiographic appearance and interventional procedures. Radiographies. 2001;21:1239-50.

10. Rowe VL, Hood DB, Liphan J, Terramini T, Torres G, Katz S. Initial experience with dorsal venous arterialization for limb salvage. Ann Vasc Surg. 2002;16:187-92.

11. Herrison C, Simon L. Le pied diabétique. Paris, Milan, Barcelona: Masson Ed; 1993. p. 5.

12. Lengua F. Le pontage d'artérialisation veineuse distale peut-il être bénéfique au pied diabétique avec nécrose? Chirurgie. 1994-1995;120:1346-52.

13. Lengua F, Cavero C, Barriga H, Zuñe A. L'artérialisation veineuse périphérique temporaire peut-elle éviter des amputations pour ischémie? Médecine et Armées. 2004;32:103108.

14. Lengua Almora F. Artérialisation du pied pour ischémie. Dernière chance avant l'amputation chez le diabétique. Lima: Delvi; 2007.

15. Leriche R. Bases de la chirurgie physiologique. Mass Ed; 1955. Préface.

16. Wahlberg E. Angiogenesis and arteriogenesis in limb ischaemia. J Vasc Surg. 2003;38:198-203.

17. Mousa AY. Angiogenesis in limb ischemia. In: Whittemore AD, editor. Advances in Vascular Surgery. 2004. p. 122.

18. Veith FJ, Weiser RK, Gupta SK, et al. Diagnosis in management of failing lower extremity arterial reconstruction prior to graft occlusion. J Cardiovasc Surg. 1984;25:381-4.

19. Isaksson L, Lundgren F. Vein bypass surgery to the foot in patients with diabetes in critical ischaemia. Br J Surg. 1994;81:517-20.

20. Pomposelli F, Maracaccio EJ, Gibbon GW, Campbell DR, Logerfo FW. Dorsalis pedis bypass durable limb salvage for foot ischaemia in patients with diabetes mellitus. J Vasc Surg. 1995; 2:375-84.

21. Panayiotopoulos YP, Reidy JF, Taylor PR. The concept of knee salvage: why does a failed femoral/pedal arterial bypass not affect the amputation level? Eur J Vasc Endovasc Surg. 1997; 13:447-85.

22. Toursarkissiam B, Shireman PK, Schoolfield J, Blumoff RI. Evolution après Occlusion d'un pontage Distal chez le Diabétique. Ann Chir Vasc. 2003;17:670-5.

23. Darling RC, Roddy SP, Chang BB, et al. Long-term results of revised infrainguinal arterial reconstruction. J Vasc Surg. 2002;35:773-8.

24. Mar CHR, Ramos G et al. Salvamento del pie diabético con la arterialización de las venas del pie. Cir Gral. 2007;29S1:135. 
25. Busato CR, Utrabo CAL, Housome JK, Gomes RZ. Arterialização do arco venoso do pé para tratamento da isquemia crítica sem leito distal. Cir Vasc Angiol. 1999;15:117-21.

26. Busato CR, Lima Utrabo CA, Zanetti Gomes R, et al. Arterialization of the venous arch of the foot for the treatment of thromboangeiitis obliterans. J Vasc Bras. 2008;7:267-71.

Correspondencia:

Servicio de Cirugía torácica y C-V, Hospital de Policía Av. Brasil s/n - Lima, Perú

E-mail: lenguafrancisco@hotmail.com

\section{Aportes de los autores}

Concepción y diseño del estudio: FLA

Análisis e interpretación de los datos: FLA

Recolección de datos: FLA, ALM, JV, CA

Redacción del artículo: FLA

Revisión crítica del texto: FLA

Aprobación final del artículo*: FLA

Análisis estadística: N/A

Responsabilidad general por el estudio: FLA

Fuentes de financiamiento: N/A

* Todos los autores leyeron y aprobaron la versión final del artículo enviada al J Vasc Bras. 